\title{
L'INVENTAIRE FORESTIER NATIONAL SUISSE 1982-1986
}

II a déjà été question dans cette revue de l'inventaire forestier national suisse $\left(n^{\circ} 4 / 1985\right.$ : " les inventaires forestiers en Suisse", par R. Schlaepfer, voir pp. 253-254).

Le premier passage des équipes sur le terrain eut lieu de 1982 à 1986. Voici maintenant la publication correspondante, qui ne peut que forcer le respect, tant par la masse, l'intérêt et la clarté des résultats chiffrés présentés au lecteur, que par la qualité hors du commun de leur présentation ${ }^{(1)}$.

Nous n'en donnerons ici qu'un bref aperçu.

\section{MÉTHODOLOGIE SOMMAIRE}

La méthodologie initiale fut l'œuvre du Professeur A. Kurt. La réalisation fut confiée à l'Institut fédéral suisse de Recherches forestières (Directeur Bosshard d'abord, puis Directeur Schlaepfer), conforté par le Service fédéral des Forêts (Directeur de Coulon).

Première phase sur photographies aériennes, après report d'un réseau maillé carré de $1 \mathrm{~km}$ de côté, échelle moyenne des photographies aériennes: 1/25000.

41291 "points" pour toute la Suisse, dont 11863 se trouvent en forêt.

L'interprétation sur photographies comporte la désignation de "repères fixes " (plusieurs pour chaque point-échantillon) devant permettre de retrouver exactement les points photos sur le terrain - et de les retrouver encore plus tard.

Suit l'inventaire au sol des placettes d'échantillonnage, qui sont des placettes permanentes, reconnues avec précision. Elles devront être retrouvées avec exactitude, et remesurées au prochain inventaire. Ces placettes sont circulaires: deux cercles concentriques; dans le plus petit - deux ares - sont mesurés tous les arbres de 12 à $36 \mathrm{~cm}$ de diamètre à hauteur d'homme; dans le plus grand - cinq ares - les arbres plus gros. Un sous-échantillon d'arbres est mesuré avec plus de précision. On y emploie un dendromètre de Christen basé sur une latte de référence de $7 \mathrm{~m}$ de longueur, permettant aussi des mesures de diamètre à $7 \mathrm{~m}$ de hauteur

(1) Schweizerisches Landesforstinventar. Resultats du premier inventaire 1982-1986 (en allemand), - Publication $n^{\circ} 305,1988$, de l'Institut fédéral suisse de Recherches forestières, $\mathrm{CH}-8903$ BIRMENSDORF.

Un volume relié $(30 \times 27 \mathrm{~cm})$. 375 pages avec 334 cartes, tableaux, ou graphiques commentés; plus un recueil annexe de 13 cartes thèmatiques de la Suisse au $1 / 800000$. 
au compas finlandais. Ce sous-échantillon permettra le calcul au bureau des fonctions de cubage. De très nombreuses informations, chiffrées ou non, sont récoltées chaque fois, soit directement, soit par questionnaire présenté au service forestier local.

L'exploitation des données se fait par mécanographie au centre de calcul de l'Institut fédéral de Recherches forestières, à l'aide d'un logiciel aussi varié que flexible, permettant de nombreuses combinaisons croisées, autorisant la sortie de tableaux très différents, avec chaque fois indication des erreurs à craindre.

\section{QUELQUES RÉSULTATS}

Le plus souvent, les résultats sont ventilés soit par régions (il en est 5 : Jura, Plateau, Préalpes, Alpes, Sud des Alpes), soit par types de forêts (il en est 12 : futaie régulière, futaie d'aspect jardinée, peuplements clairsemés en permanence, etc...). Mais bien d'autres modalités de croisement existent (tranches d'altitude, essences dominantes, types de propriétaires, etc...) et sont largement utilisées.

La Suisse compte 1186300 ha de forêts:

- taux de boisement : $28,7 \%$, ce qui est remarquable pour un pays doté de tant de hautes montagnes ;

- 687100 ha sont en futaie régulière, 105800 en futaie irrégulière, et 100100 en futaie d'aspect jardiné, etc...

- $68,5 \%$ des forêts suisses sont des forêts publiques, $31,5 \%$ seulement des forêts privées.

L'Épicéa commun est l'essence dominante sur 477300 ha. Vient ensuite le Hêtre (184 600 ha), puis le Sapin (124 200 ha), etc...

Le volume moyen sur pied, forêts "buissonnantes" exclues, est de $333 \mathrm{~m}^{3} / \mathrm{ha}$.

On retrouve là encore le classement: Épicéa d'abord, puis Hêtre et Sapin. Mais la moitié du volume sur pied de la forêt suisse est consacrée au seul Épicéa...

Les tranches d'altitude les plus boisées se situent de 1000 à 1600 mètres.

Expositions, pentes, distances de débardage, etc... les résultats de l'inventaire sont ètonnants de diversité, et d'intérêt. II y a même une carte des acidités $(\mathrm{pH})$ des sols de toutes les forêts suisses!

Manque pourtant une évaluation importante, et certains s'en étonneront: on sait bien que la forêt a fourni en moyenne au pays, de 1980 à 1985, 4,2 millions de $\mathrm{m}^{3}$ annuels de bois. Mais on ne connaît pas encore ce qu'est l'accroissement des peuplements sur pied: il faudra pour cela attendre la comparaison avec l'inventaire initial (l'actuel) que permettra le deuxième passage en inventaire, prévu à partir de 1992. La Suisse reste fidèle, au plan national comme au plan local, à la " Méthode du Contrôle" de Gurnaud et de Biolley.

C'est là un choix qui donne à réfléchir, et digne d'intérêt pour tous.

Plusieurs tentatives d'évaluation immédiate sont cependant faites, à partir des données de l'inventaire :

- de l'accroissement annuel des forêts suisses, en utilisant travaux de recherches et tables de production.

On arrive à 7 ou 8 millions de $\mathrm{m}^{3}$. 
- du potentiel d'exploitation annuel à court terme existant, en utilisant la modélisation (deux modèles très différents de sylviculture pour les prochaines années).

Le premier modèle conduit à admettre 6,7 millions de $\mathrm{m}^{3}$ annuels, et le second 6,9 millions de $\mathrm{m}^{3}$ effectivement exploitables.

Le but principal de l'inventaire forestier national suisse a été ainsi défini : le contrôle à grande échelle (großräumig) de l'évolution de la forêt suisse.

L'inventaire 1982-1986 constitue effectivement un point de départ de toute première qualité.

$$
\text { ** }
$$

En même temps que les données de l'Inventaire forestier national est présenté le rapport "Sanasilva" sur les dégâts (Waldschaden) aux forêts suisses.

Cette simultanéité ne relève pas du hasard: les travaux de l'IFN fournissent en effet les bases permettant de réaliser correctement les inventaires annuels "Sanasilva".

J. PARDÉ

\section{ERRATUM}

Parue dans l'article sur le Pin maritime des Landes du $n^{\circ} 2 / 1989$ de la Revue forestière française, la photographie de la page 134 est due non pas à M. Arbez mais à J. Timbal.

Elle représente la place d'expérience du Bray, près de Pierroton/Cestas, où depuis deux ans les chercheurs de l'INRA étudient de manière détaillée le cycle de l'eau dans l'écosystème "lande humide à Molinie " et l'écophysiologie du Pin maritime. 